Europhysics Letters

PREPRINT

\title{
Temperature dependent transport of correlated disordered electrons: elastic vs. inelastic scattering
}

\author{
M. C. O. Aguiar ${ }^{1,2}$, E. Miranda ${ }^{2}$, V. Dobrosavljević ${ }^{1}$, E. Abrahams $^{3}$ and \\ G. KOTLIAR ${ }^{3}$ \\ 1 Department of Physics and National High Magnetic Field Laboratory, Florida State \\ University - Tallahassee, FL 32306, USA \\ 2 Instituto de Física Gleb Wataghin, Unicamp - C.P. 6165, Campinas, SP 13083-970, \\ Brazil \\ 3 Center for Materials Theory, Serin Physics Laboratory, Rutgers University - 136 Frel- \\ inghuysen Road, Piscataway, NJ 08854, USA
}

PACS. 71.10.Fd - Lattice fermion models (Hubbard model, etc.).

PACS. 72.10. $-\mathrm{d}$ - Theory of electronic transport; scattering mechanisms.

PACS. $71.30 .+\mathrm{h}-$ Metal-insulator transitions and other electronic transitions.

\begin{abstract}
Temperature dependent transport of disordered electronic systems is examined in the presence of strong correlations. In contrast to what is assumed in Fermi liquid approaches, finite temperature behavior in this regime proves largely dominated by inelastic electron-electron scattering. This conclusion is valid in the strong coupling limit, where the disorder, the correlations and the Fermi energy are all comparable, as in many materials near the metal-insulator transition.
\end{abstract}

Temperature dependence of transport is well understood in ordinary metals, where it is dominated by electron-phonon scattering at room temperature. Impurity scattering [1] becomes more important close to $T=0$ (where phonons are frozen out), resulting in temperatureindependent (residual) resistivity. Weak temperature dependence in this regime reflects multiple-scattering processes leading to so-called "quantum" corrections, including weak localization and "interaction" effects [1].

Recent work [2] emphasized that these corrections reflect the interference on Friedel oscillations produced by impurities embedded in an electron gas. In this picture, temperature dependence emerges due to elastic scattering off the screened impurity potential (which is temperature dependent). The mechanism was argued [2] to apply equally well to both the ballistic and the diffusive regime. In either case, however, these processes are expected to dominate only if inelastic scattering plays a sub-dominant role.

Renewed interest in these issues has resulted from recent observations [3] of a surprisingly large drop of resistivity at low temperatures in silicon MOSFET's . Because this behavior begins to emerge already at temperatures comparable to the Fermi energy $(\sim 10 \mathrm{~K})$, estimates show [3] that it takes place in the ballistic regime. Accordingly, several authors $[2,4-6]$ have proposed that this reflects temperature dependent screening of the random potential. On the

(C) EDP Sciences 
other hand, the phenomenon is believed $[3,7]$ to occur in the strongly correlated regime, where inelastic electron-electron scattering may be equally important.

In this Letter, we address the importance of inelastic processes as a competing mechanism to temperature-dependent elastic scattering off the screened impurity potential. This is difficult within Fermi liquid approaches [1,2], which implicitly assume the sub-dominance of inelastic processes. A framework where this general question can be answered in a precise and controlled fashion is provided by Dynamical Mean Field Theory (DMFT) [8], which describes both the elastic and the inelastic processes on the same footing. Our results demonstrate that: (i) In the regime of strong correlation, where the interaction, disorder, and the Fermi energy are all comparable, there is a surprisingly large drop of resistivity (up to a factor of ten or more). (ii) Here, Fermi liquid coherence occurs only at rather low temperatures, while strong inelastic electron-electron scattering (leading to decoherence) sets in rapidly as the temperature is raised [9]. In fact, inelastic processes completely dominate the entire temperature regime where the large resistivity drop is found $\left(0.04 \leq T / E_{F} \leq 0.3\right)$.
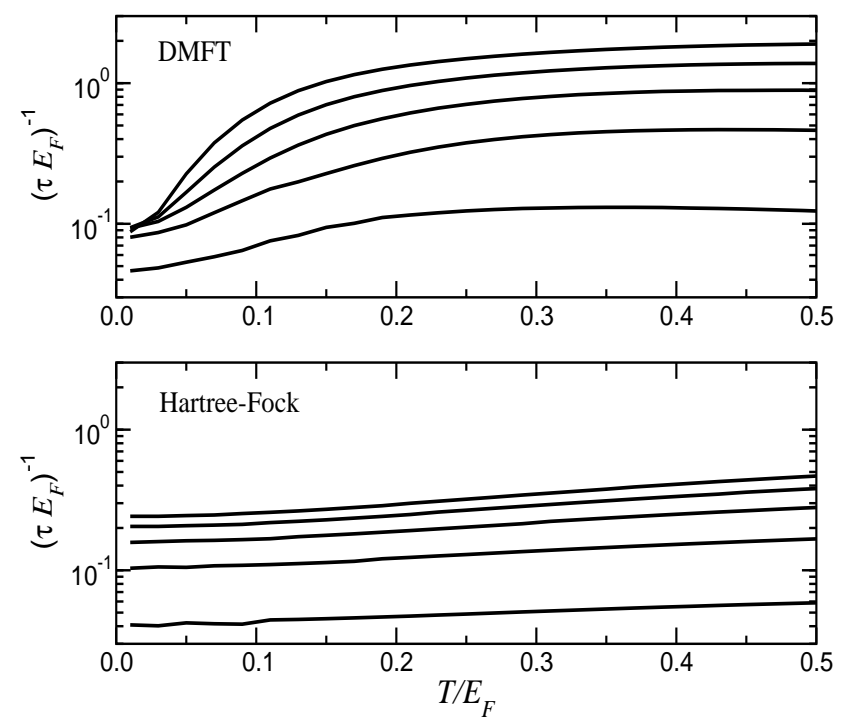

Fig. 1 - Scattering rate $\tau^{-1}(\sim$ resistivity) as a function of temperature. Results are shown for the disorder strength $W$ equal to the interaction $U$, as we reduce the Fermi energy $E_{F} / U=2.0,1.0$, $0.67,0.5,0.4$ (bottom to top curves). Note the large resistivity drop in the DMFT solution (top), but much weaker temperature dependence within the Hartree-Fock approach (bottom).

Finite temperature DMFT for disordered electrons. We considered a half-filled Hubbard model in the presence of random site energies, as given by the Hamiltonian

$$
H=-t \sum_{<i j>\sigma} c_{i \sigma}^{\dagger} c_{j \sigma}+\sum_{i \sigma} \epsilon_{i} n_{i \sigma}+U \sum_{i} n_{i \uparrow} n_{i \downarrow} .
$$

Here $c_{i \sigma}^{\dagger}\left(c_{i \sigma}\right)$ creates (destroys) a conduction electron with spin $\sigma$ on site $i, n_{i \sigma}=c_{i \sigma}^{\dagger} c_{i \sigma}$ is the particle number operator, $t$ is the hopping amplitude, and $U$ is the on-site repulsion. The random site energies $\varepsilon_{i}$ are assumed to have a uniform distribution of width $W$. Within DMFT for disordered electrons [10], a quasiparticle is characterized by a local but site-dependent [11] self-energy function $\Sigma_{i}(\omega)=\Sigma\left(\omega, \varepsilon_{i}\right)$. To calculate these self-energies, the problem is 
mapped onto an ensemble of Anderson impurity problems [10] embedded in a self-consistently calculated conduction bath. In this approach, only quantitative details of the solution depend on the details of the electronic band structure; in the following we concentrate on a semicircular model density of states. To solve DMFT equations at finite temperature, we mostly used the iterated perturbation theory (ITP) method of Kajueter and Kotliar [12]. However, we carefully checked that all the qualitative features that we report also appear when we solve DMFT equations using a Quantum Monte Carlo impurity solver [8].
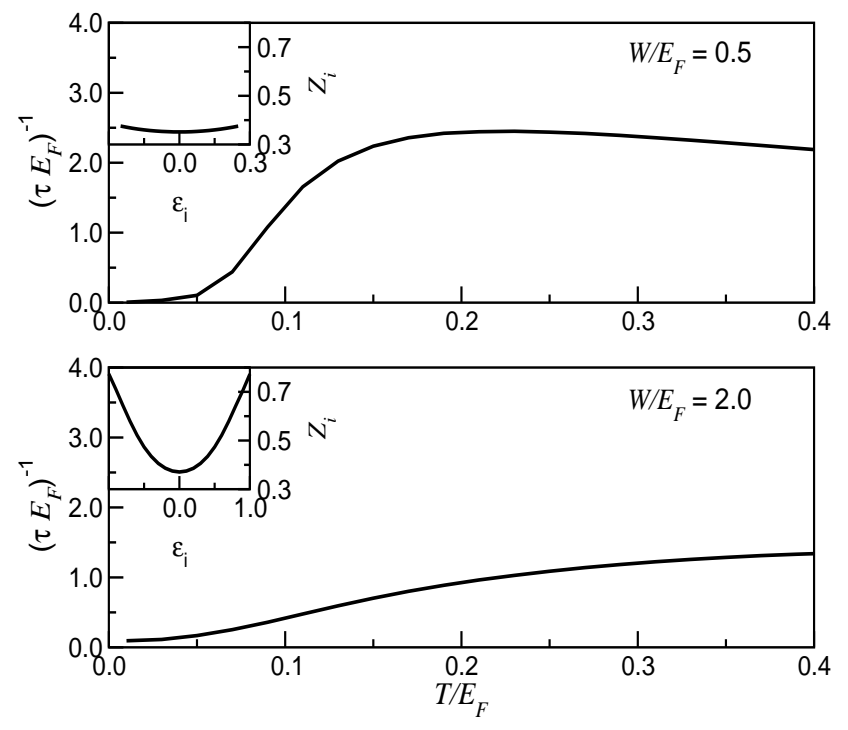

Fig. 2 - Disorder-dependence of the scattering rate for $U=2 E_{F}$, evaluated for weak $\left(W=0.5 E_{F}\right.$; top) and strong ( $W=2 E_{F}$; bottom) disorder. Insets show the distributions of local quasiparticle weights $Z_{i}$.

Temperature-dependent scattering rate. Within DMFT [8], the temperature dependence of the resistivity essentially follows that of the total scattering rate, which takes the form $\tau^{-1}=-2 \operatorname{Im} \Sigma_{a v}(\omega=0)$, where the "average" self energy [10] corresponds to the disorderaveraged local Green's function $\bar{G}(\omega)=\left\langle G_{i}(\omega)\right\rangle_{\varepsilon_{i}}=G_{o}\left[\omega-\Sigma_{a v}(\omega)\right]$, and $G_{o}(\omega)$ is the "bare" Green's function evaluated at $U=W=0$. To examine the effect of strong correlations on transport, we first concentrate on the experimentally relevant regime where the disorder and the correlations are comparable. We set $U=W$, and examine the evolution of $\tau^{-1}(T)$ as the Fermi energy is gradually reduced. Typical results of DMFT calculations are shown in fig. 1 (top). We find that, as soon as the interaction $U$ is comparable to electronic bandwidth $B$ (at half-filing $B=2 E_{F}$ ), the scattering rate displays a dramatic drop (of order ten!) below temperatures $T \sim 0.3 E_{F}$, very similar to the experiments [3]. We contrast this result to that of standard weak-coupling approaches [4-6], where the temperature dependence is much weaker and occurs over a very broad temperature range set simply by the bare Fermi scale. To make this comparison more precise, we solve our DMFT equations by using the Hartree-Fock (HF) approximation [6] where $\Sigma_{i}(\omega)=U n_{i}$; the results are shown in fig. 1] (bottom). Very weak temperature dependence is found, and one has to go to very high temperature $\left(T \gg E_{F}\right)$ to get an appreciable rise in resistivity. Note that, while giving much higher resistivity at higher temperatures, the DMFT method also produces appreciably lower 
resistivity at $T=0$, consistent with the phenomenon of correlation-enhanced screening of the random potential [13].
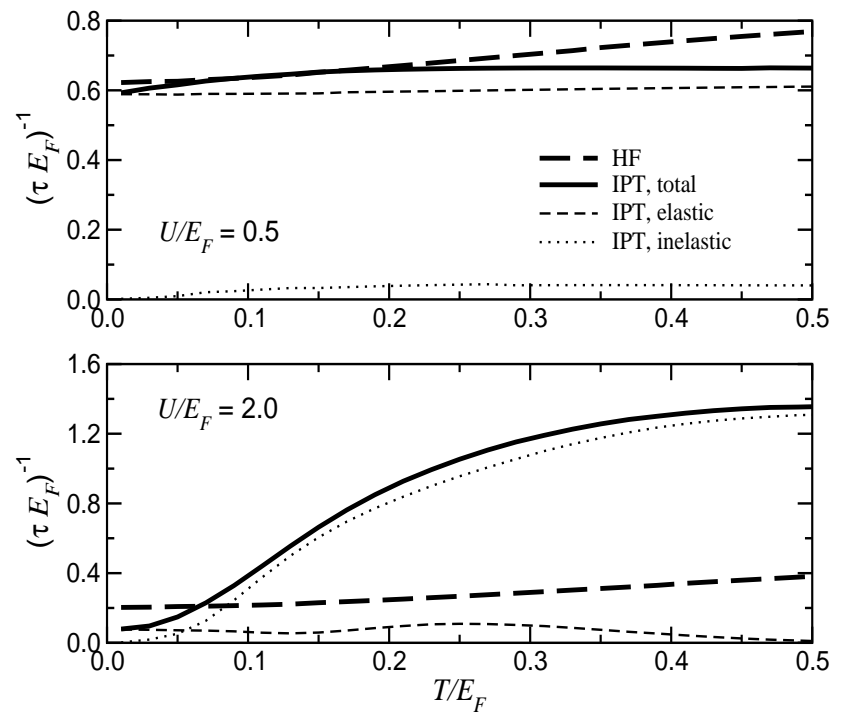

Fig. 3 - DMFT results for $W=2 E_{F}$ showing the total, elastic, and inelastic scattering rates as functions of temperature are compared to predictions of the Hartree-Fock (HF) approximation. Inelastic scattering dominates in the strongly correlated limit.

Gradual decoherence due to disorder. What sets the energy scale for the resistivity drop? To answer this, we contrast results obtained for $U=2 E_{F}$ at weak and strong disorder, as shown in fig. 2. At weak disorder $\left(W=0.5 E_{F}\right)$, the behavior is similar to the clean case [8], where a dramatic resistivity rise is found above a well defined "decoherence" temperature $T^{*} \approx 0.1 Z E_{F}[10]$, where the quasiparticles become ill-defined (here $Z$ is the quasiparticle weight, see below). This behavior is characteristic of many strongly correlated systems such as heavy fermion compounds, but such an extremely large rise is not seen in two-dimensional electron gases [3]. On the other hand, our results for the moderately disordered situation $\left(W=2 E_{F}\right)$ show a much more gradual resistivity rise, as seen in fig. 2 and in the $2 \mathrm{D}$ experiments. To understand this behavior, we note that in correlated disordered systems the quasiparticle weight becomes a strongly site-dependent $[10,11]$ quantity $Z_{i}$, which in the DMFT limit is defined by

$$
Z_{i}=\left[1-\left.\frac{\partial}{\partial \omega} \operatorname{Im} \Sigma_{i}(\omega)\right|_{\omega \rightarrow 0}\right]^{-1}
$$

The insets in fig. 2 show the distributions of $Z_{i}=Z\left(\varepsilon_{i}\right)$ for the two cases. For $U=2 E_{F}$ and weak disorder, the $Z_{i}$-s are narrowly distributed around $Z \approx 0.36$ (corresponding to a mass enhancement $m^{*} / m=Z^{-1} \approx 2.8$, and a decoherence temperature $T^{*} / E_{F} \approx 0.04$ ), giving rise to a sharply defined decoherence scale. In contrast, for stronger disorder the $Z_{i}$-s are distributed over a broad interval $0.37<Z_{i}<0.8$, corresponding to a broad distribution of local decoherence scales $T_{i}^{*} \approx 0.1 Z_{i} E_{F}$. As the temperature is raised in the presence of strong disorder, more and more sites gradually become incoherent and act as strong scattering centers. If the distribution $P\left(T_{i}^{*}\right)$ is broad, then at intermediate temperatures $T_{\min }^{*}<T<T_{\max }^{*}$ we 
expect the density of such scattering centers to grow linearly with temperature, resulting in a roughly linear resistivity in this range. This behavior is indeed observed in our calculation of the full scattering rate, which for strong disorder shows a roughly linear dependence over an appreciable range. Interestingly, the overall shape of this temperature dependence looks remarkably similar to the experimental data on silicon MOSFET's [3].
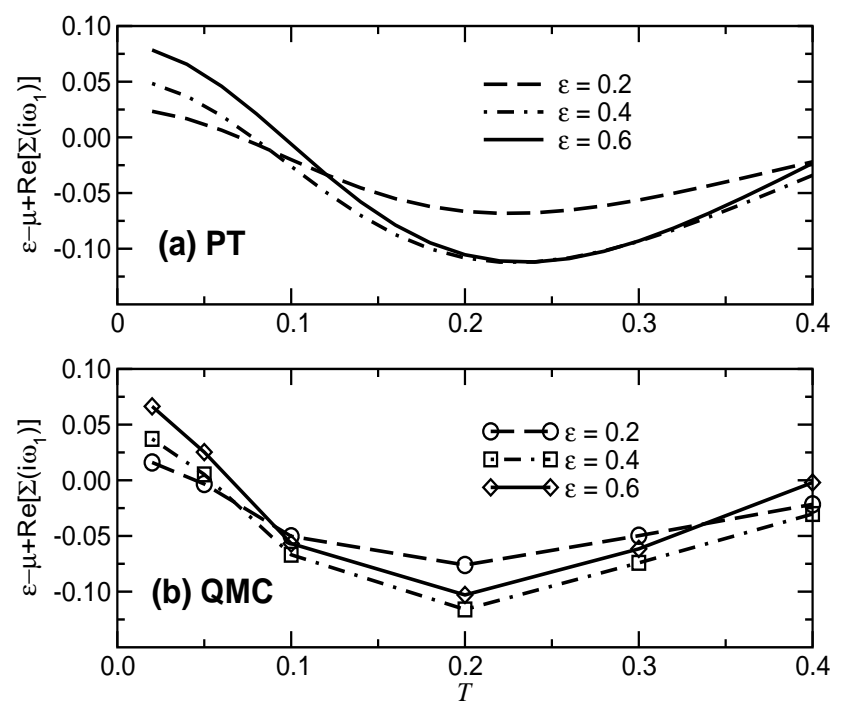

Fig. 4 - Non-monotonic temperature dependence of renormalized (screened) site energies $v_{i}(T)$ (see text) in the strongly correlated regime. Results shown correspond to a simple Anderson-impurity model with $U=3$ in a featureless host $\left(E_{F}=1\right)$, using the IPT (top) and QMC (bottom) as the impurity solver.

Elastic or inelastic scattering? The total scattering rate $\tau^{-1}$ which we have calculated describes the contribution of both the elastic and the inelastic scattering. However, to better understand which of these two processes dominates, we will separately estimate each of these contributions, as follows. Both are completely determined by the zero frequency limit of the local self-energy function, viz. $\Sigma_{i}(T)=\lim _{\omega \longrightarrow 0} \Sigma\left(\omega, \varepsilon_{i}\right)$. Its real part determines the renormalized (screened) random potential [13] $v_{i}(T)=\varepsilon_{i}+\operatorname{Re} \Sigma_{i}(T)$, while the imaginary part describes the local inelastic scattering rate $\tau_{\text {inelastic }}^{-1}(i)=-2 \operatorname{Im} \Sigma_{i}(T)$ (which is nonzero only at $T>0)$. Using our self-consistent procedure, we explicitly calculate both $\operatorname{Re} \Sigma_{i}(T)$ and $\operatorname{Im} \Sigma_{i}(T)$ at a given temperature $T$, as functions of the local site energy $\varepsilon_{i}$. Once these quantities are known, we can estimate the elastic (inelastic) contribution to the total scattering rate by simply dropping the imaginary (real) part of $\varepsilon_{i}+\Sigma_{i}(T)$, before computing $\bar{G}(\omega)$ from which $\tau^{-1}=-2 \operatorname{Im} \Sigma_{a v}(\omega=0)$ is calculated.

In this way we have (for $W=2 E_{F}$ ) computed the total, the elastic, and the inelastic scattering rates as functions of temperature, as shown in fig. 3. It is also instructive to compare our DMFT results to those obtained for the same model using the HF approximation. This weak-coupling approach is similar to those used by most other theories $[2,4-6]$, which largely ignore the inelastic scattering. We find that in the weakly interacting limit $\left(U=0.5 E_{F}\right.$; top panel) the elastic scattering dominates, and good agreement is found between DMFT and HF predictions. However, when strong correlations are present $\left(U=2 E_{F}\right.$; bottom panel), the 
inelastic scattering proves much larger then the elastic component for all except the lowest temperatures (elastic and inelastic contributions become comparable around $T / E_{F} \sim 0.04$ ). These results demonstrate that inelastic scattering dominates over the entire temperature range where the large resistivity drop is found, in striking contrast to weak-coupling predictions $[2,4-6]$. In this regime, the elastic scattering component has an extremely weak and even non monotonic temperature dependence, and clearly has very little physical content if considered in absence of inelastic processes.

To clarify this issue, we have explicitly computed the temperature dependence of the renormalized site energies $v_{i}(T)$. In the weakly interacting limit, these quantities are found to have a modest and monotonic temperature dependence in agreement with HF predictions. However, in the regime of strong correlations, we find surprising non-monotonic temperature dependence where for some values of $\varepsilon_{i}$, and at intermediate temperatures, negative screening is found $\left(v_{i}(T)<0\right.$ for $\left.\varepsilon_{i}>0\right)$. We have examined this puzzling behavior in great detail, and have found that this is a very general feature of strongly correlated systems, which depends only weakly on the specific self-consistency condition used.

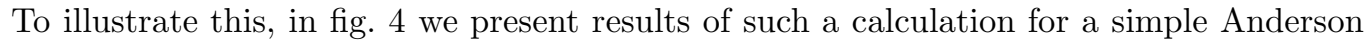
impurity model with bare site energy $\varepsilon_{i}$ and on-site repulsion $U$, embedded in a featureless (semicircular) electron bath. To demonstrate that this behavior is not an artifact of our IPT impurity solver, we present results of both IPT and numerically exact QMC calculations for the same model, which produce almost identical results.

We emphasize that the energy scale associated with the $v_{i}(T)$-s is very small in the intermediate temperature range where negative screening emerges. In this regime, the scattering is completely dominated by inelastic processes, so this puzzling behavior has by itself very little physical consequence. Nevertheless, these results clearly indicate that theories which ignore inelastic scattering are very likely to produce unreliable and even physically incorrect results if used in the incoherent regime where Fermi liquid theory cannot be applied.

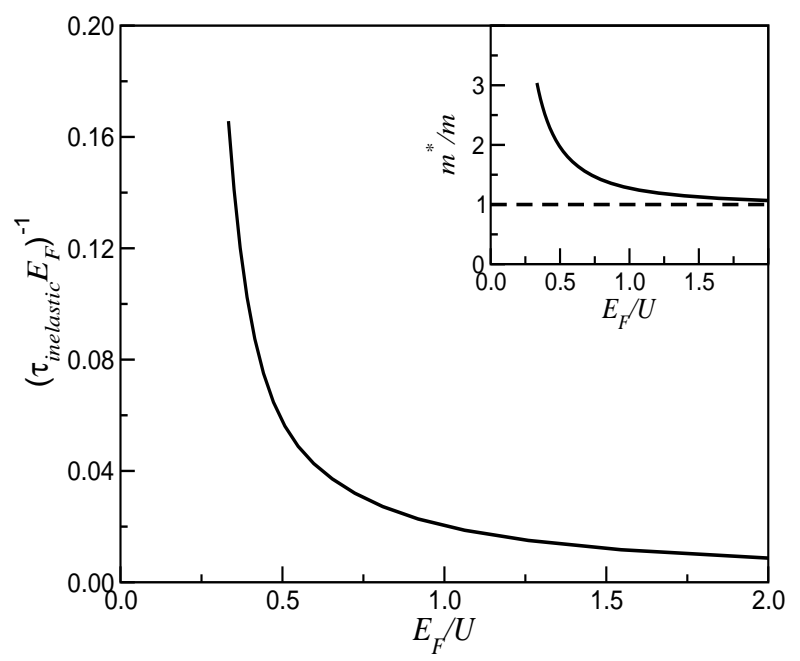

Fig. 5 - Inelastic scattering rate for $W=U$ and $T / E_{F}=0.05$, as a function of $E_{F} / U$. The inset shows the (average) effective mass enhancement $m^{*} / m=\left\langle Z_{i}\right\rangle^{-1}$ in the same range of parameters. Large enhancement of dephasing is found in the same range where the mass enhancement is large, similarly as in experiments. 
Enhanced dephasing in the strongly correlated regime. In fig. 3, we showed the inelastic scattering rate as a function of temperature. We have also computed it as a function of density (i.e. $\left.E_{F} / U\right)$ at $T / E_{F}=0.05$ and $W=U$. As shown in fig. 5 the inelastic scattering rate becomes appreciable in the same range where the effective mass $\left(m^{*} / m \sim\langle Z\rangle^{-1}\right.$; see inset) is enhanced, and we enter the regime of strong correlations. This prediction awaits experimental tests on sufficiently homogeneous samples.

In summary, we have presented quantitatively reliable model calculations for correlated disordered electrons in the strong coupling limit where the disorder strength, the interactions, and the Fermi energy are all comparable. Our results demonstrate that inelastic electronelectron scattering dominates the regimes relevant to many puzzling experimental situations. We expect large resistivity drops, similar to what we find, to also occur in 3D situations when correlations are sufficiently large, and diagonal disorder not too strong [14]. In fact, in many 3D weakly disordered heavy-fermion compounds, even larger resistivity drops are observed below a coherence temperature. Our DMFT approach, while being able to address the nontrivial interplay of disorder and strong correlations is nevertheless too simple to include localization effects that are important closer to the metal-insulator transition. These can be incorporated in our framework using recently developed extensions [15] of DMFT, but this problem remains a fascinating direction for future work.

We thank M.J. Rozenberg for providing us with the QMC code for the Hubbard model, and A. Georges, S. Das Sarma, D. Popović for useful discussions. This work was supported by FAPESP 99/00895-9 (MCOA), 01/00719-8 and CNPq 301222/97-5 (EM), and NSF grants DMR-9974311 and DMR-0234215 (VD), DMR-9976665 (EA), and DMR-0096462 (GK). VD and GK also thank KITP at UCSB (NSF grant PHY99-07949) where part of this work was carried out.

\section{REFERENCES}

[1] Lee P. A. and Ramakrishnan T. V., Rev. Mod. Phys., 57 (1985) 287.

[2] Zala G. et al., Phys. Rev. B, 64 (2001) 214204.

[3] Abrahams E. et al., Rev. Mod. Phys., 73 (2001) 251.

[4] Das Sarma S. et al., Phys. Rev. Lett., 83 (1999) 164.

[5] Dolgopolov V. T. and Gold A., JETP Lett., 71 (2000) 27.

[6] Herbut I. F., Phys. Rev. B, 63 (2001) 113102.

[7] Dobrosavluević V. et al., Phys. Rev. Lett., 90 (2003) 016402.

[8] Georges A. et al., Rev. Mod. Phys., 68 (1999) 13.

[9] This feature is common to all theories of metals with strong correlation.

[10] Dobrosavluević V. and Kotliar G., Phys. Rev. Lett., 71 (1993) 3218; Phys. Rev. B, 50 (1994) 1430.

[11] The fact that the quasiparticle parameters are site-dependent was emphasized by: ZimANYI G. T. and Abrahams E., Phys. Rev. Lett., 64 (1990) 2719.

[12] Kajueter H. et al., Phys. Rev. Lett., 77 (1996) 131.

[13] Tanasković D. et al., Phys. Rev. Lett., 91 (2003) 066603.

[14] The well-studied MIT in Si:P does not meet the mentioned criteria since there one is not close to the clean Mott transition. In addition, Si:P has rather strong off-diagonal disorder that is not moderated by the screening effects which are important in our calculation.

[15] Dobrosavljević V. and Kotliar G., Phys. Rev. Lett., 78 (1997) 3943; Miranda E. and Dobrosavluević V., Phys. Rev. Lett., 86 (2001) 264. 